\title{
The phenotypic relationship of Neisseria polysaccharea to commensal and pathogenic Neisseria spp.
}

\author{
KATHRYN J. CANN and T. R. ROGERS \\ Department of Medical Microbiology, Charing Cross and Westminster School, 17 Horseferry Road, London \\ SW1
}

\begin{abstract}
Summary. Eight of 22 non-capsulate strains of Neisseria meningitidis previously isolated from primary school children were re-identified as $N$. polysaccharea by aminopeptidase reactions and polysaccharide production. $N$. polysaccharea was not identified amongst 91 non-capsulate strains of $N$. meningitidis isolated from adults attending the Genito-urinary Medicine clinic, Westminster Hospital, London. The biochemical reactions of $N$. polysaccharea strains were similar to those of $N$. lactamica and $N$. gonorrhoeae, but $N$. polysaccharea could be distinguished from these organisms by examination of $\beta$-galactosidase activity, carbohydrate reactions and polysaccharide production. Sodium dodecyl sulphate-polyacrylamide gel electrophoresis revealed closer similarity of $N$. polysaccharea to $N$. lactamica than to the pathogenic Neisseria spp. An additional finding was variation in the position of one of the major proteins of $N$. lactamica in the 34-39-Kda region.
\end{abstract}

\section{Introduction}

Riou et al. (1983) first proposed the new species Neisseria polysacchareae, later renamed $N$. polysaccharea (Guibourdenche et al., 1986), after investigating approximately 3500 strains of referred Neisseria and Branhamella spp. Thirteen isolates, from the throats of healthy children, could not be speciated and were subsequently designated $N$. polysaccharea, a species which could be differentiated from $N$. meningitidis by the absence of glutamyl aminopeptidase activity, polysaccharide production and cysteine requirement for growth on Catlin medium. Bouquete et al. (1986) subsequently identified $50 \mathrm{~N}$. polysaccharea strains from 216 noncapsulate strains of $N$. meningitidis received in a meningococcus reference laboratory $(23 \%)$, they also investigated 102 non-capsulate meningococcal isolates from a survey of children aged 5-9 years (Saez-Nieto et al., 1985) of which 38 (37\%) were identified as $N$. polysaccharea. In this study we examined strains of $N$. meningitidis obtained from a previous survey of primary school children, aged 4-11 years (Cann et al., 1987), as well as isolates obtained from adults attending the Genito-urinary Medicine clinic, Westminster Hospital, in order to assess the prevalence of $N$. polysaccharea strains in adults and children. We also examined the relation-

Received 22 July 1988; revised version accepted 15 Jan. 1989. ship between $N$. polysaccharea and $N$. lactamica which is a common commensal of the nasopharynx in young children.

\section{Materials and methods}

\section{Isolates}

A total of 251 strains was examined: 22 non-capsulate isolates of $N$. meningitidis were obtained from a previous survey of nasopharyngeal carriage in primary school children undertaken because of a cluster of cases of invasive meningococcal disease caused by a serogroup $B$, 15P1.16 strain (Cann et al., 1987); 91 meningococcal strains were collected during 1 year from adult patients attending the Genito-urinary Medicine clinic; 138 strains of $N$. lactamica were also collected from the study of primary school children. All strains were isolated from the posterior nasopharynx and stored in $10 \%$ glycerol peptone at $-70^{\circ} \mathrm{C}$. The 113 isolates were identified as $N$. meningitidis by conventional laboratory methods: they were gram-negative, oxidase-positive cocci which were negative for $\beta$-galactosidase (ONPG) and produced acid from the oxidation of glucose and maltose but not sucrose or lactose. $N$. lactamica strains were identified by a positive ONPG reaction. Strains were serogrouped by slide agglutination with Wellcome meningococcal agglutinating sera against groups A, B, C, W135, X, Y, Z. Serotyping was performed by co-agglutination with monoclonal antibodies generously supplied by Drs W. Zollinger and J. Poolman. The serotyping antibodies used were P1.2, 3, 9, 15 and 16, 2a, 2b, 2c and 15. 


\section{Biochemical tests}

$\gamma$-L-Glutamyl and L-hydroxyproline aminopeptidase activity was measured by a non-quantitative modification of the method described by Ison et al. (1982). A heavy suspension of each organism was made in saline and assayed in duplicate for both enzymes in a flat-bottomed microtitration plate (Dynatech). A strain of $N$. meningitidis $\mathrm{B}, 15 \mathrm{P} 1.16$ and a $N$. lactamica strain were included as controls. Those isolates that gave negative results for $\gamma$-L-glutamyl aminopeptidase were subcultured on nutrient agar (Oxoid) containing horse serum 5\% and dextrose $5 \%$ at $36^{\circ} \mathrm{C}$ in reduced oxygen tension (i.e., in a candle jar) with $N$. meningitidis $\mathrm{B}, 15 \mathrm{P} 1.16$ and an $N$. lactamica strain as controls. Polysaccharide production was detected by the addition of a drop of Gram's iodine solution (potassium iodide $2 \%$, iodine $1 \%$ ) to a colony which became black immediately if positive (personal communication, J. Knapp, Center for Disease Control, Atlanta). This test could also be performed on the growth on the sucrose slope from the initial carbohydrate identification tests. Those isolates that gave negative results for $\gamma$-L-glutamyl aminopeptidase were subcultured at $22^{\circ} \mathrm{C}$ and $36^{\circ} \mathrm{C}$, on Thayer Martin agar, in reduced oxygen tension, with $N$. meningitidis and $N$. lactamica as controls.

\section{$S D S-P A G E$}

The 22 non-capsulate meningococcal isolates plus a group of $\mathrm{B}, 15 \mathrm{P} 1.16$ isolates from children, 20 meningococcal isolates of different sero-groups and sero-types from adults and $20 \mathrm{~N}$. lactamica strains were prepared for sodium dodecyl sulphate-polyacrylamide gel electrophoresis (SDS-PAGE). Each isolate was inoculated into Tryptone Soya Broth (Oxoid) and incubated overnight, at $36^{\circ} \mathrm{C}$ on a gyratory shaker at $150 \mathrm{rpm}$. The growth was centrifuged to obtain a cell pellet which was washed in saline, re-centrifuged and resuspended in saline. The protein concentration of the suspension was estimated by the method of Lowry et al. (1951) and diluted with saline to a concentration of $2 \mu \mathrm{g} / \mu \mathrm{l}$. The standardised cell suspensions were then diluted 1 in 2 with sample buffer (SDS $4 \%$, mercaptoethanol $10 \%$, glycerol $20 \%$ in $0.125 \mathrm{M}$ Tris/HCl). Electrophoresis of these samples was performed with $12.5 \%$ polyacrylamide gels in a discontinuous buffer system (Laemmli, 1970) for $1 \mathrm{~h}$. The following mol.-wt standards (Kda) were applied: bovine serum albumin 66 ; ovalbumin 45 ; pepsin 34.7 ; trypsinogen 24 ; $\beta$-lactoglobulin 18.4; and lysozyme 14.3. Gels were stained with Brilliant Blue (Sigma Chemical Company, P.O. Box 14508, St Louis, MO 63178, USA) and dried with a slab gel drier (LKB, 232 Addington Road, Selsdon, S. Croydon CR2 8YD). Two gonococcal lysates, Kellogg strain F62 and strain G7 (Johnston et al., 1976) were provided by $\mathrm{Dr} \mathrm{C}$. Ison as controls.

\section{Results}

Table I shows the reactions distinguishing $N$. meningitidis, $N$. polysaccharea, $N$. lactamica and $N$. gonorrhoeae. Eight isolates from children but none of the adult strains showed the characteristics of $N$. polysaccharea. Our previous study had been performed as three cross sectional surveys within a 1year period. Seven of these eight isolates were from different children with ages ranging from 4 to 11 years (one isolate was carried by the same child in two surveys). Seven were from the school which had suffered cases of meningococcal disease and one was from the smaller control school. This difference in the number of $N$. polysaccharea isolated from each school was not statistically significant. The eight $N$. polysaccharea strains and all $138 \mathrm{~N}$. lactamica strains were ungroupable and non-typable. The eight $N$. polysaccharea strains also failed to grow at $22^{\circ} \mathrm{C}$, on Thayer Martin agar, in reduced oxygen tension.

Table I. Characteristics discriminating $N$. meningitidis, $N$. polysaccharea, $N$. lactamica and $N$. gonorrhoeae

\begin{tabular}{|c|c|c|c|c|}
\hline \multirow[b]{2}{*}{ Test } & \multicolumn{4}{|c|}{ Results obtained with } \\
\hline & N. meningitidis & $N$. polysaccharea & N. lactamica & $N$. gonorrhoeae \\
\hline \multicolumn{5}{|l|}{ Acid from } \\
\hline glucose & + & + & + & + \\
\hline maltose & + & + & + & - \\
\hline lactose & - & - & + & - \\
\hline sucrose & - & - & - & - \\
\hline $\begin{array}{l}\beta \text {-Galactosidase } \\
\quad \text { activity (ONPG) }\end{array}$ & & & & \\
\hline $\begin{array}{l}\text { activity (ONPG) } \\
\gamma \text {-L-Glutamyl aminopeptidase }\end{array}$ & - & - & + & - \\
\hline activity & + & - & - & - \\
\hline L-Hydroxyproline aminopeptidase & & & & \\
\hline activity & - & + & + & + \\
\hline Polysaccharide production & - & + & - & - \\
\hline
\end{tabular}




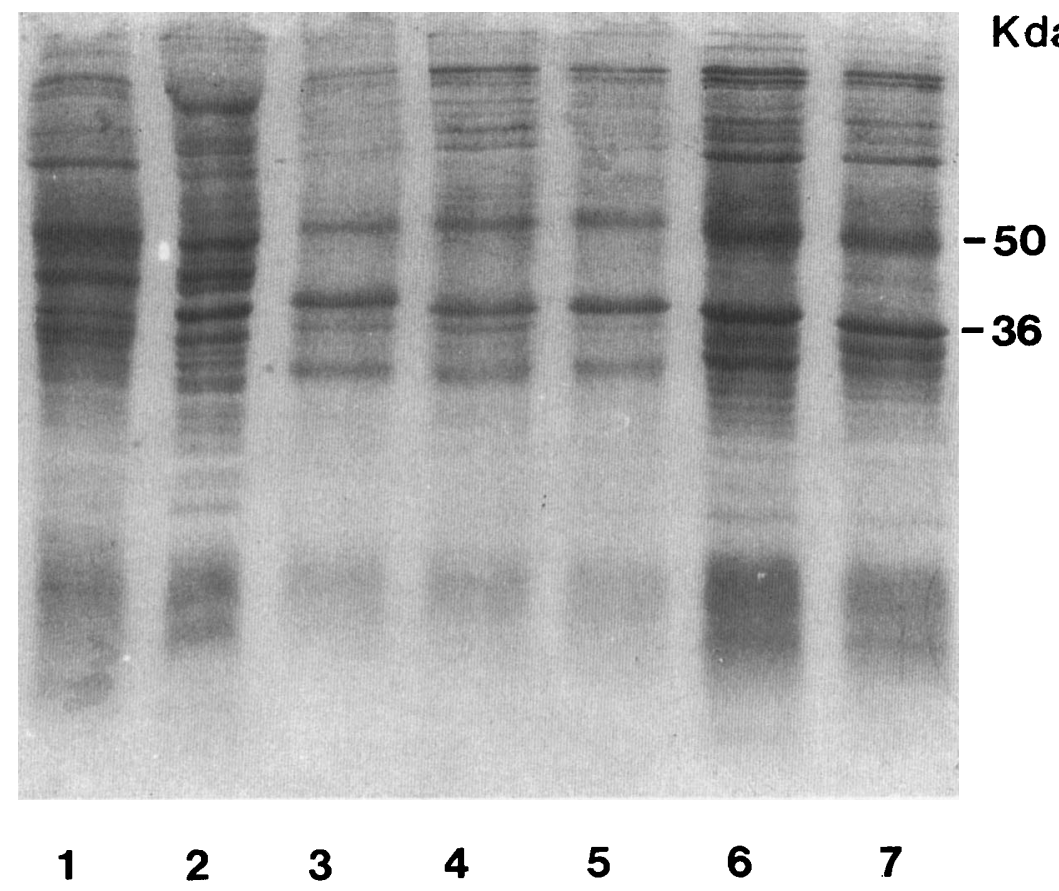

Figure. SDS-PAGE of Neisseria spp. Lanes 1 and 2, N. meningitidis B, 15 P1. 16 and ungroupable, non-typable, respectively; lanes 3 and 4, N. lactamica; lane 5, N. polysaccharea; lanes 6 and 7, N. gonorrhoeae.

The figure shows the SDS-PAGE protein profiles of $N$. meningitidis $\mathrm{B}, 15 \mathrm{P} 1.16$ and an ungroupable non-typable meningococcus, $N$. polysaccharea, $N$. lactamica, and $N$. gonorrhoeae. The selection of $N$. meningitidis strains examined showed a variety of SDS-PAGE types as described by Mocca and Frasch (1982). The pattern of bands appears to be very similar in all four species in the high mol. wt region, $60-82 \mathrm{Kda}$. The presence of a major protein band in the $48-50-\mathrm{Kda}$ region was seen in $N$. lactamica and $N$. polysaccharea, similar to $N$. meningitidis. In $N$. gonorrhoeae a major band was seen in a slightly lower position-44 Kda. Both $N$. lactamica and $N$. polysaccharea produce simpler protein patterns with a second major band seen at $34-36 \mathrm{Kda}$. In $N$. lactamica strains we observed variation in position of this protein band. Twenty $N$. lactamica strains were examined, of which 11 had a $34-36-\mathrm{Kda}$ band but the other nine had a major band at 37-39-Kda. By this technique, $N$. polysaccharea appears to be more closely related to $N$. lactamica than to either $N$. meningitidis or $N$. gonorrhoeae.

\section{Discussion}

We chose to examine meningococcal strains for $\mathrm{L}$-hydroxyproline aminopeptidase as well as $\gamma$-L- glutamyl aminopeptidase and $\beta$-galactosidase as a result of the work of D'Amato et al. (1978), which showed that profiles based on these three enzymes were the most useful in distinguishing Neisseria spp. $N$. polysaccharea, like $N$. lactamica and other commensal Neisseria spp., is positive for L-hydroxyproline aminopeptidase. $N$. gonorrhoeae is also L-hydroxyproline aminopeptidase-positive; however, on SDS-PAGE $N$. polysaccharea appears to be more similar to $N$. lactamica.

DNA relatedness studies have shown that $N$. gonorrhoeae, $N$. meningitidis, $N$. lactamica and $N$. polysaccharea form a single genospecies within which the four sub-species can be delineated (Guibourdenche et al., 1986). Clinically, however, it is more useful to regard them as separate species with the meningococcus and gonococcus frequently acting as pathogens whereas $N$. polysaccharea and $N$. lactamica appear to be non-pathogenic and to be found more frequently in the nasopharynx of children than adults.

The similarities observed on SDS-PAGE between $N$. polysaccharea and $N$. lactamica, and between these two species and $N$. meningitidis, give some support to the theory that carriage of commensal Neisseria spp. may contribute to the acquisition of immunity to $N$. meningitidis. $N$. polysaccharea and $N$. lactamica have been shown to 
cross-react with anti-meningococcal sera, especially of group B (Gold et al., 1978; Saez-Nieto et al., 1985; Boquete et al., 1986) but the precise nature of the role of these two organisms in the acquisition of immunity to meningococcal disease requires further study.

Confusion of $N$. polysaccharea strains with $N$. meningitidis will lead to falsely high estimates of meningococcal carriage and acquisition in a population. In our study of primary school children, the true meningococcal carriage and acquisition rates were, in fact, lower, although this does not affect the conclusions drawn. Our finding of approxi-

\section{REFERENCES}

Bouquete M T, Marcos C, Saez-Nieto J A 1986 Characterization of Neisseria polysacchareae sp. nov. (Riou, 1983) in previously identified noncapsular strains of Neisseria meningitidis. Journal of Clinical Microbiology 23: 973-975.

Cann K J, Rogers T R, Jones D M, Noah N D, Burns C 1987 Neisseria meningitidis in a primary school. Archives of Disease in Childhood 62: 1113-1117.

D'Amato R F, Eriquez L A, Tomfohrde K M, Singerman E 1978 Rapid identification of Neisseria gonorrhoeae and Neisseria meningitidis by using enzymatic profiles. Journal of Clinical Microbiology 7: 77-81.

Gold R, Goldschneider I, Lepow M L, Draper T F, Randolph M 1978 Carriage of Neisseria meningitidis and Neisseria lactamica in infants and children. Journal of Infectious Diseases 137: 112-121.

Guibourdenche M, Popoff M Y, Riou J Y 1986 Deoxyribonucleic acid relatedness among Neisseria gonorrhoeae, $N$. meningitidis, $N$. lactamica, N. cinerea and "Neisseria polysaccharea". Annales de l'Institut Pasteur, Microbiology 37B: 177-185.

Ison C, Glynn A A, Bascomb S 1982 Acquisition of new genes mately one third (8 out of 22) of non-capsulate strains of "meningococci" from young children displaying the characteristics of $N$. polysaccharea is similar that of Boquete et al. (1986) who studied a similar age group. The ease with which $N$. polysaccharea can be distinguished from $N$. meningitidis by testing for polysaccharide production should prevent future confusion and overestimates of meningococcal carriage rates.

We are grateful to Drs D. M. Jones and C. Ison for helpful discussions and also to Dr J. Knapp for advice on the polysaccharide production test. K.J.C. was supported by a grant from the North West Thames Regional Health Authority.

by oral Neisseria. Journal of Clinical Pathology 35: 11531157.

Johnston K H, Holmes K K, Gotschlich E C 1976 The serological classification of Neisseria gonorrhoeae. I. Isolation of the outer membrane complex responsible for serotypic specificity. Journal of Experimental Medicine 143: 741-758.

Laemmli U K 1970 Cleavage of structural proteins during the assembly of the head of bacteriophage T4. Nature 227:680685.

Lowry O H, Roseborough N J, Farr A L, Randall R J 1951 Protein measurement with the folin phenol reagent. Journal of Biological Chemistry 193: 265-275.

Mocca L F, Frasch C E 1982 Sodium dodecyl sulfate polyacrylamide gel typing system for characterization of Neisseria meningitidis isolates. Journal of Clinical Microbiology 16: 240-244.

Riou J Y, Guibourdenche M, Popoff M Y 1983 A new taxon in the genus Neisseria. Annales de Microbiologie 134B: 257267.

Saez-Nieto J A et al. 1985 Carriage of Neisseria meningitidis and Neisseria lactamica in a school population during an epidemic period in Spain. Journal of Hygiene 94: 279-288. 